\title{
Dependence of critical current of spin transfer torque-driven magnetization dynamics on free layer thickness
}

\author{
Tomohiro Taniguchi ${ }^{1,2}$ and Hiroshi Imamura ${ }^{1 *}$ \\ ${ }^{1}$ Nanotechnology Research Institute, National Institute of Advanced Industrial Science and Technology, \\ Central 2, 1-1-1, Umezono, Tsukuba, Ibaraki 305-8568, Japan \\ 2 Institute of Applied Physics, University of Tsukuba, Tsukuba, Ibaraki 305-8573, Japan
}

(Dated: April 23, 2022)

\begin{abstract}
The dependence of the critical current of spin transfer torque-driven magnetization dynamics on the free-layer thickness was studied by taking into account both the finite penetration depth of the transverse spin current and spin pumping. We showed that the critical current remains finite in the zero-thickness limit of the free layer for both parallel and anti-parallel alignments. We also showed that the remaining value of the critical current of parallel to anti-parallel switching is larger than that of anti-parallel to parallel switching.
\end{abstract}

PACS numbers: Valid PACS appear here

Spin transfer torque (STT)-driven magnetization dynamics is a promising technique to operate spinelectronics devices such as a non-volatile magnetic random access memory (MRAM) and a microwave generator [1, 2]. STT is the torque due to the transfer of the transverse (perpendicular to magnetization) spin angular momentum from the conducting electrons to the magnetization of the ferromagnetic metal. One of the most important quantities of STT-driven magnetization dynamics is the critical current over which the dynamics of the magnetization is induced. The typical value of the critical current density is on the order of $10^{6}-10^{8}$ $\left[\mathrm{A} / \mathrm{cm}^{2}\right][3,4,5]$. Control of the value of the critical current is required to reduce the energy consumption of spin-electronics devices.

In Slonczewski's theory of STT [1], the critical current of P-to-AP (AP-to-P) switching is expressed as [6, 7]

$$
I_{\mathrm{c}}^{\mathrm{P} \rightarrow \mathrm{AP}(\mathrm{AP} \rightarrow \mathrm{P})}=\frac{2 e M S d}{\hbar \gamma \eta_{\mathrm{P}(\mathrm{AP})}} \alpha_{0} \omega_{\mathrm{P}(\mathrm{AP})},
$$

where $e$ is the absolute value of the electron charge, $\hbar$ is the Dirac constant, and $M, \gamma, S, d$ and $\alpha_{0}$ are the magnetization, gyromagnetic ratio, cross section area, thickness and the intrinsic Gilbert damping constant of the free layer, respectively [5]. $\omega_{\mathrm{P}(\mathrm{AP})}$ is the angular frequency of the magnetization around the equilibrium point. The coefficient $\eta_{\mathrm{P}, \mathrm{AP}}$ characterizes the strength of STT, and depends only on the relative angle of the magnetizations of the fixed and free layer [1, 6, 7]. According to Eq. (1), the critical current vanishes in the zero-thickness limit of the free layer, $d \rightarrow 0$.

However, recently, Chen et al. [5] reported that the critical current of STT-driven magnetization dynamics of a CPP-GMR spin valve remains finite even in the zerothickness limit of the free layer. What are missed in the above naive considerations based on Slonczewski's theory are the effects of the finite penetration depth of the

*Corresponding author. Email address: h-imamura@aist.go.jp

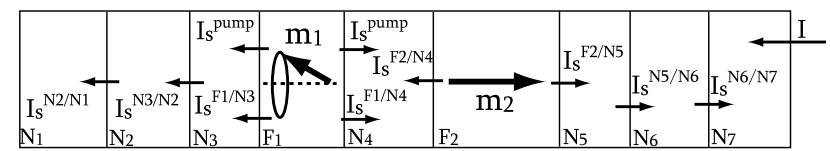

FIG. 1: The schematic view of the nonmagnetic(N) / ferromagnetic $(\mathrm{F})$ multilayer. $I$ and $\mathbf{I}_{s}^{\text {pump }}$ are the electric current and pumped spin current, respectively. $\mathbf{I}_{s}^{\mathrm{N}_{i}\left(\mathrm{~F}_{k}\right) / \mathrm{N}_{j}}$ is the spin current induced by the spin accumulations in each layer. $\mathbf{m}_{k}(k=1,2)$ is the unit vector pointing the direction of the magnetization of the $\mathrm{F}_{k}$ layer.

transverse spin current, $\lambda_{\mathrm{t}}, 8,8,9,10$ and of spin pumping [11, 12, 13, 14]. We investigated the critical current of STT-driven magnetization switching from AP to $\mathrm{P}$ alignment by taking into account both the finite penetration depth of the transverse spin current and the spin pumping, and showed that the critical current remains finite in the zero-thickness limit of the free layer [15]. We also showed that the remaining value of the critical current is mainly determined by spin pumping. Although our results [15] agree well with the experimental results of Chen et al. [5], we investigated only the critical current of AP-to-P switching, $I_{\mathrm{c}}^{\mathrm{AP} \rightarrow \mathrm{P}}$. For the manipulation of spin-electronics devices, the thickness dependence of the critical current of P-to-AP switching, $I_{\mathrm{c}}^{\mathrm{P} \rightarrow \mathrm{AP}}$, should also be investigated.

In this paper, we study the critical current of STTdriven magnetization switching both from $\mathrm{P}$ to AP alignment and from AP to $\mathrm{P}$ alignment by taking into account both the finite penetration depth of the transverse spin current and the spin pumping. We show that both critical currents, $I_{\mathrm{c}}^{\mathrm{P} \rightarrow \mathrm{AP}}$ and $I_{\mathrm{c}}^{\mathrm{AP}} \rightarrow \mathrm{P}$, remain finite in the zero-thickness of the free layer. We also show that $I_{\mathrm{c}}^{\mathrm{P} \rightarrow \mathrm{AP}}$ is larger than $I_{\mathrm{c}}^{\mathrm{AP}} \rightarrow \mathrm{P}$ over the whole range of the free layer thickness, and thus, the remaining value of $I_{\mathrm{c}}^{\mathrm{P} \rightarrow \mathrm{AP}}$ is larger than that of $I_{\mathrm{c}}^{\mathrm{AP} \rightarrow \mathrm{P}}$. The difference between the remaining values of the critical currents, $I_{\mathrm{c}}^{\mathrm{P} \rightarrow \mathrm{AP}}$ and $I_{\mathrm{c}}^{\mathrm{AP}} \rightarrow \mathrm{P}$, can be explained by considering how the strength of STT, $\eta$, depends on the magnetic alignment.

A schematic view of the system we consider is shown 
in Fig. 1. Two ferromagnetic layers $\left(\mathrm{F}_{1}\right.$ and $\left.\mathrm{F}_{2}\right)$ are sandwiched by the nonmagnetic layers $\mathrm{N}_{i}(i=1-7)$. The $\mathrm{F}_{1}$ and $\mathrm{F}_{2}$ layers correspond to the free and fixed layers, respectively. $\mathbf{m}_{k}(k=1,2)$ is the unit vector pointing in the direction of the magnetization of the $\mathrm{F}_{k}$ layer. $I$ is the electric current flowing perpendicular to the film plane.

The electric current and pumped spin current at the $\mathrm{F}_{k} / \mathrm{N}_{i}$ interface (into $\mathrm{N}_{i}$ ) is obtained by using the circuit theory [12, 16]:

$$
\begin{aligned}
I^{\mathrm{F}_{k} / \mathrm{N}_{i}} & =\frac{e g}{2 h}\left[2\left(\mu_{\mathrm{F}_{k}}-\mu_{\mathrm{N}_{i}}\right)+p \mathbf{m}_{k} \cdot\left(\boldsymbol{\mu}_{\mathrm{F}_{k}}-\boldsymbol{\mu}_{\mathrm{N}_{i}}\right)\right], \\
\mathbf{I}_{s}^{\text {pump }} & =\frac{\hbar}{4 \pi}\left(g_{\mathrm{r}}^{\uparrow \downarrow} \mathbf{m}_{1} \times \frac{\mathrm{d} \mathbf{m}_{1}}{\mathrm{~d} t}+g_{\mathrm{i}}^{\uparrow \downarrow} \frac{\mathrm{d} \mathbf{m}_{1}}{\mathrm{~d} t}\right),
\end{aligned}
$$

where $h=2 \pi \hbar$ is the Planck constant, $g=g^{\uparrow \uparrow}+g^{\downarrow \downarrow}$ is the sum of the spin-up and spin-down conductances, $p=\left(g^{\uparrow \uparrow}-g^{\downarrow \downarrow}\right) /\left(g^{\uparrow \uparrow}+g^{\downarrow \downarrow}\right)$ is the spin polarization of the conductances, and $g_{\mathrm{r}(\mathrm{i})}$ is the real (imaginary) part of the mixing conductance. $\mu_{\mathrm{N}_{i}, \mathrm{~F}_{k}}$ and $\boldsymbol{\mu}_{\mathrm{N}_{i}, \mathrm{~F}_{k}}$ are the charge and spin accumulation, respectively. The spin current at each $\mathrm{F}_{k} / \mathrm{N}_{i}$ and $\mathrm{N}_{i} / \mathrm{N}_{j}$ interface (into $\mathrm{N}_{i}$ ) is given by [10, 16]

$$
\begin{aligned}
\mathbf{I}_{s}^{\mathrm{F}_{k} / \mathrm{N}_{i}} & =\frac{1}{4 \pi}\left[g\left\{p\left(\mu_{\mathrm{F}_{k}}-\mu_{\mathrm{N}_{i}}\right)+\frac{1}{2} \mathbf{m}_{k} \cdot\left(\boldsymbol{\mu}_{\mathrm{F}_{k}}-\boldsymbol{\mu}_{\mathrm{N}_{i}}\right)\right\} \mathbf{m}_{k}\right. \\
& -g_{\mathrm{r}}^{\uparrow \downarrow} \mathbf{m}_{k} \times\left(\boldsymbol{\mu}_{\mathrm{N}_{i}} \times \mathbf{m}_{k}\right)-g_{\mathrm{i}}^{\uparrow \downarrow} \boldsymbol{\mu}_{\mathrm{N}_{i}} \times \mathbf{m}_{k} \\
& \left.+t_{\mathrm{r}}^{\uparrow \downarrow} \mathbf{m}_{k} \times\left(\boldsymbol{\mu}_{\mathrm{F}_{k}} \times \mathbf{m}_{k}\right)+t_{\mathrm{i}}^{\uparrow \downarrow} \boldsymbol{\mu}_{\mathrm{F}_{k}} \times \mathbf{m}_{k}\right] \\
\mathbf{I}_{s}^{\mathrm{N}_{i} / \mathrm{N}_{j}} & =-\frac{g_{\mathrm{N}_{i} / \mathrm{N}_{j}}}{4 \pi}\left(\boldsymbol{\mu}_{\mathrm{N}_{i}}-\boldsymbol{\mu}_{\mathrm{N}_{j}}\right)
\end{aligned}
$$

where $t_{\mathrm{r}(\mathrm{i})}^{\uparrow \downarrow}$ is the real (imaginary) part of the transmission mixing conductance at the $\mathrm{F}_{k} / \mathrm{N}_{i}$ interface and $g_{\mathrm{N}_{i} / \mathrm{N}_{j}}$ is the conductance of the one spin channel at the $\mathrm{N}_{i} / \mathrm{N}_{j}$ interface.

The spin accumulations in the $\mathrm{N}$ and $\mathrm{F}$ layer obey the diffusion equation [8, 10, 17]. The spin accumulation in the $\mathrm{N}$ layer, $\boldsymbol{\mu}_{\mathrm{N}}$, decays exponentially with the spin diffusion length $\lambda_{\mathrm{sd}(\mathrm{N})}$. The longitudinal and transverse spin accumulations in the $\mathrm{F}$ layer are defined as $\left(\mathbf{m} \cdot \boldsymbol{\mu}_{\mathrm{F}}\right) \mathbf{m}$ and $\mathbf{m} \times\left(\boldsymbol{\mu}_{\mathrm{F}} \times \mathbf{m}\right)$, respectively. The longitudinal and transverse spin accumulations decay exponentially with the spin diffusion length $\lambda_{\mathrm{sd}\left(\mathrm{F}_{\mathrm{L}}\right)}$ and with the penetration depth of the transverse spin current $\lambda_{\mathrm{t}}$, respectively.

The total spin currents across the $\mathrm{N}_{3} / \mathrm{F}_{1}$ and $\mathrm{F}_{1} / \mathrm{N}_{4}$ interfaces, i.e., $\mathbf{I}_{s}^{(1)}=\mathbf{I}_{s}^{\text {pump }}+\mathbf{I}_{s}^{\mathrm{F}_{1} / \mathrm{N}_{3}}$ and $\mathbf{I}_{s}^{(2)}=\mathbf{I}_{s}^{\text {pump }}+\mathbf{I}_{s}^{\mathrm{F}_{1} / \mathrm{N}_{4}}$, exert the torque $\boldsymbol{\tau}=\mathbf{m}_{1} \times\left[\left(\mathbf{I}_{s}^{(1)}+\mathbf{I}_{s}^{(2)}\right) \times \mathbf{m}_{1}\right]$ on the magnetization $\mathbf{m}_{1}$. In order to obtain the spin current $\mathbf{I}_{s}^{(1,2)}$, we solve the diffusion equation of spin accumulation in each layer. The boundary conditions are as follows. We assume that the thicknesses of the $\mathrm{N}_{1}$ and $\mathrm{N}_{7}$ layer are much larger than their spin diffusion length, and that the spin current is zero at the outer boundary of the $\mathrm{N}_{1}$ and $\mathrm{N}_{7}$ layer. We also assume that the spin current is continuous at all interfaces and that the electric current is constant through the entire structure.

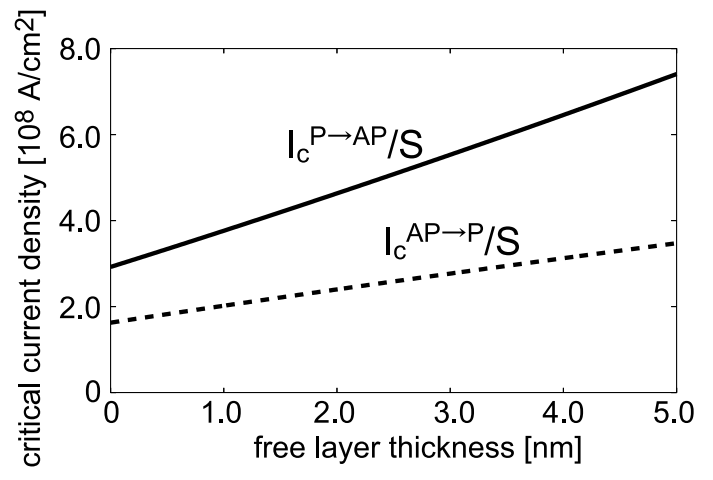

FIG. 2: The critical current densities of P-to-AP switching $\left(I_{\mathrm{c}}^{\mathrm{P} \rightarrow \mathrm{AP}} / S\right)$ and AP-to-P switching $\left(I_{\mathrm{c}}^{\mathrm{AP} \rightarrow \mathrm{P}} / S\right)$ in STTdriven magnetization dynamics are shown against the free layer thickness.

The torque $\boldsymbol{\tau}$ modifies the Landau-Lifshitz-Gilbert (LLG) equation of magnetization $\mathbf{m}_{1}$ as [12]

$$
\begin{aligned}
\frac{\mathrm{d} \mathbf{m}_{1}}{\mathrm{~d} t}= & -\gamma \mathbf{m}_{1} \times \mathbf{B}_{\mathrm{eff}}+\frac{\gamma}{M S d} \boldsymbol{\tau}+\alpha_{0} \mathbf{m}_{1} \times \frac{\mathrm{d} \mathbf{m}_{1}}{\mathrm{~d} t} \\
& =-\gamma_{\mathrm{eff}} \mathbf{m}_{1} \times \mathbf{B}_{\mathrm{eff}}+\frac{\gamma_{\mathrm{eff}}}{\gamma}\left(\alpha_{0}+\alpha^{\prime}\right) \mathbf{m}_{1} \times \frac{\mathrm{d} \mathbf{m}_{1}}{\mathrm{~d} t},
\end{aligned}
$$

where $\mathbf{B}_{\text {eff }}$ is the effective magnetic field, and $\alpha^{\prime}=$ $\alpha_{c}+\alpha_{\text {pump }}$ is the enhancement of the Gilbert damping constant. The enhancement $\alpha_{c}$ is proportional to the electric current and independent of the pumped spin current. The enhancement $\alpha_{\text {pump }}$ represents the contribution from the pumped spin current and is independent of the electric current. The enhancement of the gyromagnetic ratio, $\gamma_{\mathrm{eff}} / \gamma$, is a function of both the electric current and the pumped spin current.

The critical current of the STT-driven magnetization dynamics is defined by the electric current that satisfies the condition, $\alpha_{0}+\alpha_{c}+\alpha_{\text {pump }}=0$, and given by

$$
I_{\mathrm{c}}^{\mathrm{P} \rightarrow \mathrm{AP}(\mathrm{AP} \rightarrow \mathrm{P})}=\frac{2 e M S d}{\hbar \gamma \tilde{\eta}_{\mathrm{P}(\mathrm{AP})}}\left(\alpha_{0}+\alpha_{\mathrm{pump}}\right) \omega_{\mathrm{P}(\mathrm{AP})},
$$

where the coefficient $\tilde{\eta}_{\mathrm{P}, \mathrm{AP}}$ characterizes the strength of STT due to the electric current, and is determined by the diffusion equations of the spin accumulations. Thus, $\tilde{\eta}_{\mathrm{P}, \mathrm{AP}}$ is the function of $d / \lambda_{\mathrm{sd}\left(\mathrm{F}_{\mathrm{L}}\right)}, d / \lambda_{\mathrm{t}}$ and the relative angle of the magnetizations of the $\mathrm{F}_{1}$ and $\mathrm{F}_{2}$ layers.

We performed numerical calculation to obtain the critical currents $I_{\mathrm{c}}^{\mathrm{P} \rightarrow \mathrm{AP}}$ and $I_{\mathrm{c}}^{\mathrm{AP} \rightarrow \mathrm{P}}$. The system consists of nine layers as shown in Fig. 1 where $\mathrm{F}_{1}$ and $\mathrm{F}_{2}$ are Co, $\mathrm{N}_{1}, \mathrm{~N}_{3}, \mathrm{~N}_{4}, \mathrm{~N}_{5}$ and $\mathrm{N}_{7}$ are $\mathrm{Cu}$, and $\mathrm{N}_{2}$ and $\mathrm{N}_{6}$ are Pt. The thicknesses of the $\mathrm{N}_{3}, \mathrm{~N}_{4}$ and $\mathrm{N}_{5}$ layers are $10 \mathrm{~nm}$, the thicknesses of the $\mathrm{N}_{2}$ and $\mathrm{N}_{6}$ layers are $3 \mathrm{~nm}$ and the thickness of the $\mathrm{F}_{2}$ layer is $12 \mathrm{~nm}[5]$. The thickness of the $\mathrm{N}_{1}$ and $\mathrm{N}_{7}$ layers are taken to be $10 \mu \mathrm{m}$. The spin diffusion length of $\mathrm{Cu}$ and $\mathrm{Pt}$ are 1000 and $14 \mathrm{~nm}$, respectively [18]. The conductance at the $\mathrm{Cu} / \mathrm{Pt}$ interface is $35 \mathrm{~nm}^{-2}$ [18]. The magnetization, the intrinsic Gilbert damping constant and the gyromagnetic ratio of Co are 


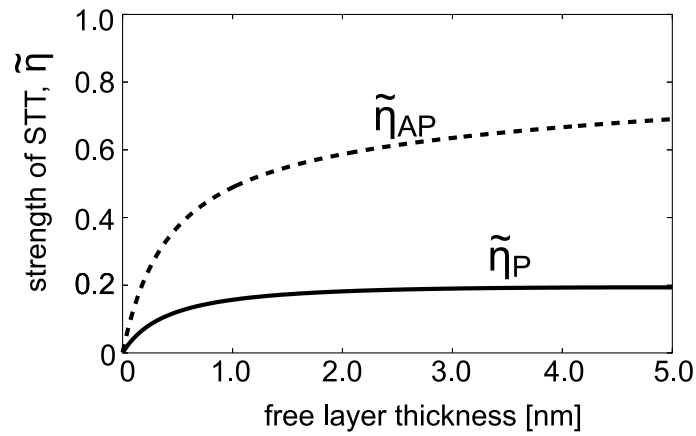

FIG. 3: The coefficient $\tilde{\eta}$ in the $\mathrm{P}$ state $\left(\tilde{\eta}_{\mathrm{P}}\right)$ and AP state $\left(\tilde{\eta}_{\mathrm{AP}}\right)$, against the free layer thickness.

$0.14 \mathrm{~T}, 0.008$ and $1.89 \times 10^{11} \mathrm{~Hz} / \mathrm{T}$, respectively 5, 19]. The polarization $p$ is taken to be 0.46 for Co [18]. The spin diffusion length of Co is $40 \mathrm{~nm}$ [18]. The penetration depth of the transverse spin current of Co is $4.2 \mathrm{~nm}$ [9, 15]. The conductances at the $\mathrm{Co} / \mathrm{Cu}$ interface, $g / S$, $g_{\mathrm{r}}^{\uparrow \downarrow} / S$ and $g_{\mathrm{i}}^{\uparrow \downarrow} / S$, are 50,27 and $0.4 \mathrm{~nm}^{-2}$, respectively [12, 13, 16]. We assume that $t_{\mathrm{r}}=t_{\mathrm{i}}$ where $t_{\mathrm{r}, \mathrm{i}} / S$ at the $\mathrm{Co} / \mathrm{Cu}$ interface is taken to be $6.0 \mathrm{~nm}^{-2}$. The angular frequency is $\omega_{\mathrm{P}(\mathrm{AP})}=\gamma\left[B_{\mathrm{appl}}-(+) 4 \pi M\right]$ where the strength of the applied magnetic field $B_{\text {appl }}$ is $7 \mathrm{~T}[5]$.

Figure 2 shows the dependence of the critical current density of Eq. (7) for P-to-AP switching, $I_{\mathrm{c}}^{\mathrm{P} \rightarrow \mathrm{AP}} / S$, and AP-to-P switching, $I_{\mathrm{c}}^{\mathrm{AP} \rightarrow \mathrm{P}} / S$, on the free layer thickness, d. As shown in Fig. 2, both $I_{\mathrm{c}}^{\mathrm{P} \rightarrow \mathrm{AP}}$ and $I_{\mathrm{c}}^{\mathrm{AP} \rightarrow \mathrm{P}}$ remain finite in the zero-thickness limit of the free layer. We show that the critical current $I_{\mathrm{c}}^{\mathrm{P} \rightarrow \mathrm{AP}}$ is larger than $I_{\mathrm{c}}^{\mathrm{AP} \rightarrow \mathrm{P}}$ over the whole range of the free layer thickness, and thus, the remaining value of $I_{\mathrm{c}}^{\mathrm{P} \rightarrow \mathrm{AP}}$ is larger than that of $I_{\mathrm{c}}^{\mathrm{AP} \rightarrow \mathrm{P}}$. As shown in Ref. [15], the remaining value of the critical current is mainly determined by spin pumping. It should be noted that the magnitude of the enhancement of the Gilbert damping constant due to spin pumping, $\alpha_{\text {pump }}$, is the same for both P-to-AP switching and AP-to-P switching [13, 14]. Thus, the fact that the remaining values $I_{\mathrm{c}}^{\mathrm{P} \rightarrow \mathrm{AP}}$ and $I_{\mathrm{c}}^{\mathrm{AP} \rightarrow \mathrm{P}}$ are different from each other implies that the strength of STT, $\tilde{\eta}_{\mathrm{P}, \mathrm{AP}}$, depends on the alignment of the magnetizations. As shown in Fig. 3, $\tilde{\eta}_{\mathrm{P}, \mathrm{AP}}$ decreases with a decreasing free layer thickness. On the other hand, the number of localized magnetic moments in the free layer, and therefore the STT per magnetic moment, is inversely proportional to the free layer thickness $d$. According to Eq. (7), the remaining value of the critical current is proportional to $\left(\tilde{\eta}_{\mathrm{P}, \mathrm{AP}} / d\right)^{-1}$ with $d \rightarrow 0$, where $\tilde{\eta}_{\mathrm{P}} / d \simeq 0.44 \mathrm{~nm}^{-1}$ and $\tilde{\eta}_{\mathrm{AP}} / d \simeq 1.47 \mathrm{~nm}^{-1}$ in the limit of $d \rightarrow 0$ are estimated by Fig. 3. Thus, the remaining value of $I_{\mathrm{c}}^{\mathrm{P} \rightarrow \mathrm{AP}}$ is larger than that of $I_{\mathrm{c}}^{\mathrm{AP} \rightarrow \mathrm{P}}$.

In summary, we studied the critical current of STTdriven magnetization dynamics by taking into account the finite penetration depth of the transverse spin current and spin pumping for both $\mathrm{P}$ and $\mathrm{AP}$ magnetic alignments. We showed that the critical current remains finite in the zero thickness limit of the free layer for both $\mathrm{P}$ to-AP and AP-to-P switching. We also showed that the critical current for P-to-AP switching is larger than that for AP-to-P switching over the whole range of the free layer thickness.

The authors would like to acknowledge the valuable discussions they had with K. Matsushita, J. Sato and N. Yokoshi. This work was supported by JSPS and NEDO.
[1] J. C. Slonczewski, J. Magn. Magn. Mater. 159, L1 (1996).

[2] L. Berger, Phys. Rev. B 54, 9353 (1996).

[3] S. I. Kiselev, J. C. Sankey, I. N. Krivorotov, N. C. Emley, R. J. Schoelkopf, R. A. Buhrman, and D. C. Ralph, Nature 425, 380 (2003).

[4] T. Seki, S. Mitani, K. Yakushiji, and K. Takanashi, Appl. Phys. Lett. 89, 172504 (2006).

[5] W. Chen, M. J. Rooks, N. Ruiz, J. Z. Sun, and A. D. Kent, Phys. Rev. B 74, 144408 (2006).

[6] J. Z. Sun, Phys. Rev. B 62, 570 (2000).

[7] J. Grollier, V. Cros, H. Jaffrés, A. Hamzic, J. M. George, G. Faini, J. B. Youssef, H. L. Gall, and A. Fert, Phys. Rev. B 67, 174402 (2003).

[8] S. Zhang, P. M. Levy, and A. Fert, Phys. Rev. Lett. 88, 236601 (2002).

[9] J. Zhang, P. M. Levy, S. Zhang, and V. Antropov, Phys. Rev. Lett. 93, 256602 (2004).

[10] T. Taniguchi, S. Yakata, H. Imamura, and Y. Ando,
Appl. Phys. Express 1, 031302 (2008).

[11] S. Mizukami, Y. Ando, and T. Miyazaki, Phys. Rev. B 66, 104413 (2002).

[12] Y. Tserkovnyak, A. Brataas, and G. E. W. Bauer, Phys. Rev. B 66, 224403 (2002).

[13] Y. Tserkovnyak, A. Brataas, and G. E. W. Bauer, Phys. Rev. B 67, 140404(R) (2003).

[14] T. Taniguchi and H. Imamura, Phys. Rev. B 76, 092402 (2007).

[15] T. Taniguchi and H. Imamura, Phys. Rev. B 78, 224421 (2008).

[16] A. Brataas, Y. V. Nazarov, and G. E. W. Bauer, Eur. Phys. J. B 22, 99 (2001).

[17] T. Valet and A. Fert, Phys.Rev.B 48, 7099 (1993).

[18] J. Bass and W. P. P. Jr, J. Phys.: Condens. Matter 19, 183201 (2007).

[19] J.-M. L. Beaujour, W. Chen, A. D. Kent, and J. Z. Sun, J. Appl. Phys. 99, 08N503 (2006). 